Relato de Caso

Case Report

Karina Krähembühl Salvador ${ }^{1}$

Tatiane Cristina Pereira ${ }^{1}$

Tamyne Ferreira Duarte de Moraes ${ }^{1}$

Mariana Sodário $\mathrm{Cruz}^{2}$

Mariza Ribeiro Feniman ${ }^{3}$

Descritores

Audição

Perda auditiva unilateral

Percepção auditiva

Localização de som

Questionários

Keywords

Hearing

Hearing loss, unilateral Auditory perception Sound localization

Questionnaires

Endereço para correspondência:

Mariza Ribeiro Feniman

Al. Octávio Pinheiro Brisola, 9/75, Vila Universitária, Bauru(SP), Brasil, CEP:17012-901.

E-mail: feniman@usp.br

Recebido em: 12/4/2011

Aceito em: 25/8/2011

\section{Processamento auditivo na perda auditiva unilateral: relato de caso}

\section{Auditory processing in unilateral hearing loss: case report}

\section{RESUMO}

A perda auditiva unilateral representa grande risco para o atraso acadêmico, a comunicação, o desenvolvimento social e também para o processamento auditivo. Assim, o objetivo deste estudo foi avaliar as habilidades auditivas de localização, fechamento, figura-fundo, resolução temporal e ordenação temporal simples de um sujeito do gênero masculino, 17 anos de idade, com diagnóstico de perda auditiva sensorioneural unilateral de grau profundo, de causa idiopática, sem outros comprometimentos. O processo de avaliação constituiu da aplicação de um questionário, da realização da avaliação audiológica clínica convencional (audiometria tonal, logoaudiometria e imitanciometria) e de testes de processamento auditivo monóticos (SSI ipsilateral, Teste de fala filtrada) e dióticos (Localização sonora, Memória auditiva para sons verbais, Memória auditiva para sons não verbais, AFT-R). Apenas o teste de Localização sonora apresentou resultados alterados. Não foram relatadas queixas relacionadas à habilidade de localização sonora, atenção, discriminação e compreensão auditiva. No caso em estudo, a perda auditiva sensorioneural unilateral de grau profundo não pareceu restringir o desenvolvimento das habilidades do processamento auditivo avaliadas, exceto no que se refere à localização da fonte sonora.

\begin{abstract}
Unilateral hearing loss represents great risk to academic backwardness, communication, social development, and also to auditory processing. Thus, the aim of this study was to evaluate the auditory abilities of localization, closing, figure-ground, temporal resolution, and simple temporal ordering in a male 17-year-old individual diagnosed with profound unilateral sensorineural hearing loss of idiopathic etiology, without other alterations. The evaluation process consisted on the application of a checklist, and the conduction of conventional clinical audiological evaluation (pure-tone audiometry, logoaudiometry, and tympanometry), and of monotic (ipsilateral SSI, Filtered speech test) and diotic (Sound localization, Auditory memory for verbal and non-verbal sounds, AFT-R) auditory processing test. Results showed alteration only in the sound localization test. No complaints were reported regarding the abilities of sound localization, attention, discrimination, and comprehension. In this case study, the profound unilateral sensorineural hearing loss did not seem to restrict the development of the auditory processing abilities evaluated, except for the localization of the sound source.
\end{abstract}

Trabalho realizado no Hospital de Reabilitação de Anomalias Craniofaciais, Universidade de São Paulo - USP - Bauru (SP), Brasil.

(1) Programa de Pós-Graduação (Mestrado) em Fonoaudiologia, Faculdade de Odontologia de Bauru, Universidade de São Paulo - USP - Bauru (SP), Brasil.

(2) Programa de Pós-Graduação (Doutorado) em Saúde Coletiva, Faculdade de Medicina de Botucatu, Universidade Estadual Paulista - UNESP - Botucatu(SP), Brasil.

(3) Departamento de Fonoaudiologia, Faculdade de Odontologia de Bauru, Universidade de São Paulo - USP - Bauru (SP), Brasil. 


\section{INTRODUÇÃO}

Investigações têm demonstrado vantagens da audição binaural em relação à monoaural, no que se refere à localização da fonte sonora, fenômeno de somação, eliminação do efeito sombra da cabeça, melhora do reconhecimento de fala em presença de ruído e menor esforço para ouvir ${ }^{(1,2)}$. Assim, a perda auditiva unilateral é alvo de diversos estudos, uma vez que representa grande risco para o atraso acadêmico, a comunicação, o desenvolvimento social e também para o processamento auditivo ${ }^{(3-5)}$.

A avaliação das habilidades do processamento auditivo por procedimentos padronizados tem contribuído para a identificação e intervenção das alterações encontradas. Testes de processamento auditivo, como o Pediatric Speech Intelligibility e o teste Gaps-in-Noise (GIN) foram utilizados em estudo $^{(4)}$ para avaliar, respectivamente, as habilidades auditivas de figura-fundo e resolução temporal de um grupo de 19 sujeitos (com média de idade de 12 anos), em sua maioria com perda auditiva unilateral de grau profundo, iniciada em fase préescolar, de etiologia desconhecida ou identificada. Os resultados foram comparados aos de um grupo controle pareados conforme gênero, idade e escolaridade. A autora identificou resultados inferiores no grupo com perda auditiva unilateral.

Em estudo ${ }^{(5)}$ realizado com 26 sujeitos (13 com perda auditiva unilateral e 13 com audição normal) utilizando uma bateria de testes da avaliação do processamento auditivo (localização sonora em cinco direções, teste de fala com ruído, memória sequencial verbal e não verbal, Random Gap Detection Test RGDT), a pesquisadora concluiu que a habilidade de resolução temporal não distinguiu sujeitos com e sem perda auditiva e que sujeitos com perda auditiva unilateral apresentaram pior desempenho em tarefas de localização sonora e fechamento auditivo.

Considerando os importantes efeitos de uma perda auditiva unilateral, é fundamental determinar a existência do comprometimento de habilidades auditivas, uma vez que esta informação poderá nortear as estratégias do tratamento. Por isso, julgou-se necessário identificar possíveis habilidades auditivas alteradas em um sujeito com perda auditiva unilateral.

Portanto, este trabalho teve o objetivo de avaliar as habilidades auditivas de localização, fechamento, figura-fundo, resolução temporal e ordenação temporal de um sujeito com perda auditiva unilateral sensorioneural de grau profundo.

\section{APRESENTAÇÃO DO CASO CLÍNICO}

O estudo foi aprovado pelo Comitê de Ética em Pesquisa da Faculdade de Odontologia de Bauru, Universidade de São Paulo, sob o número 023/2003. O responsável pelo sujeito em estudo assinou o Termo de Consentimento Livre e Esclarecido (TCLE), consentindo, desta forma, com a realização e divulgação desta pesquisa e seus resultados conforme Resolução 196/96 da Comissão Nacional de Ética em Pesquisa (CONEP).

O sujeito D.R.C. (gênero masculino, funileiro, 17 anos), chegou à clínica de Fonoaudiologia com a queixa de não escutar em uma das orelhas. O processo de avaliação constituiu, primeiramente, da aplicação de um questionário, visando conhecer a história auditiva do paciente. Em seguida foi realizada uma bateria de testes audiológicos: avaliação audiológica convencional (audiometria tonal liminar, logoaudiometria e imitanciometria) e os testes comportamentais do processamento auditivo (monóticos e dióticos)

Testes monóticos são testes nos quais estímulos, diferentes ou não, são apresentados simultaneamente na mesma orelha, ou seja, ipsilateralmente. Os testes selecionados foram: SSI em Português (Synthetic Sentence Identification) ${ }^{(6)}$, em escuta monótica, que avalia a habilidade de figura-fundo para sons verbais e tem por objetivo fornecer informações sobre o mecanismo fisiológico auditivo de reconhecimento de sons verbais nas relações fala/ruído zero, -10 e -15; Teste de Fala Filtrada ${ }^{(6)}$, que consiste na apresentação de uma lista 25 estímulos verbais distorcidos e avalia a habilidade de fechamento auditivo.

Os testes dióticos são testes em que estímulos iguais são apresentados simultaneamente para as duas orelhas. Os testes selecionados foram: Teste de localização sonora em cinco direções (direita, esquerda, atrás, acima e à frente) ${ }^{(6)}$, Testes de memória para sons verbais e não verbais em sequência ${ }^{(6)}$, e Teste da Fusão Auditiva-Revisado - AFT-R ${ }^{(7)}$ (procedimento para medir a habilidade do processamento temporal, determinando a duração, em milissegundos, em que o ouvinte pode detectar um breve intervalo de silêncio entre dois tons).

O teste de localização sonora (LS) avalia a habilidade auditiva de localização sonora e o mecanismo fisiológico de interação binaural ${ }^{(8)}$. Utilizando um guizo, foram pesquisadas cinco posições de localização em relação à cabeça do sujeito, de olhos vendados: à direita, à esquerda, acima, à frente e atrás. $\mathrm{O}$ sujeito deveria indicar o local do som. Para este procedimento era esperado que o sujeito acertasse pelo menos quatro das cinco direções apresentadas ${ }^{(6)}$.

No Teste de memória para sons verbais em sequência (TMSV) foram apresentadas oralmente ao sujeito três diferentes sequências de três sílabas (PA, TA, CA), que deveriam ser repetidas por ele na sequência exata. Era esperado o acerto de duas ou mais sequências. No teste de memória para sons não verbais em sequência (TMSnV) foram utilizados quatro instrumentos sonoros (guizo, coco, agogô e sino), percutidos em três diferentes sequências. O sujeito deveria apontar os instrumentos musicais de acordo com a ordem apresentada. Era esperado que o sujeito acertasse pelo menos duas sequências de quatro sons em três tentativas. Os testes denominados TMSV e TMSnV têm por objetivo fornecer informações dos mecanismos fisiológicos auditivo de processamento temporal, visando avaliar as habilidades auditivas de ordenação temporal ${ }^{(6,8)}$.

Os testes LS, TMSV e TMSnV foram demonstrados ao sujeito anteriormente a sua aplicação. Todos foram realizados em cabina acústica. Para os testes monóticos e para o AFT-R, utilizou-se o audiômetro de dois canais acoplado a um CD player.

De acordo com os dados do questionário, o paciente relatou apresentar dificuldades de atenção, localização, discriminação e compreensão auditiva, com restrição para a execução de algumas tarefas e determinadas atividades de interação social. Não foram relatadas complicações referentes aos aspectos otológicos em ambas as orelhas. $O$ paciente não soube especificar 
Tabela 1. Valores dos limiares tonais aéreos obtidos pelo paciente em estudo

\begin{tabular}{lcccccccc}
\hline Orelha & $250 \mathrm{~Hz}$ & $500 \mathrm{~Hz}$ & $1 \mathrm{kHz}$ & $2 \mathrm{kHz}$ & $3 \mathrm{kHz}$ & $4 \mathrm{kHz}$ & $6 \mathrm{kHz}$ & $8 \mathrm{kHz}$ \\
\hline $\mathrm{OD}(\mathrm{dB})$ & 5 & 10 & 10 & 10 & 10 & 15 & 15 & 10 \\
$\mathrm{OE}(\mathrm{dB})$ & 100 & 100 & 105 & 115 & $\downarrow(120)$ & $\downarrow(120)$ & $\downarrow(110)$ & $\downarrow(105)$ \\
\hline
\end{tabular}

Legenda: $\mathrm{OD}=$ orelha direita; $\mathrm{OE}=$ orelha esquerda; $\mathrm{dB}=$ decibel; $\mathrm{Hz}=$ Hertz

se a perda auditiva foi adquirida ou congênita, porém afirmou que a apresentava desde a infância.

A avaliação audiológica básica (Tabela 1) revelou limiares tonais aéreos dentro dos padrões de normalidade na orelha direita e limiares tonais aéreos rebaixados na orelha esquerda, indicando perda auditiva sensorioneural de grau profundo. Os dados da avaliação logoaudiométrica foram compatíveis com a audiometria tonal liminar. Na imitanciometria, foi observado timpanograma tipo A nas duas orelhas, indicando ausência de patologia de orelha média. Em relação aos reflexos estapedianos, houve presença de reflexos ipsilaterais e contralaterais na orelha direita e ausência de ambos à esquerda, dados compatíveis com o quadro de perda auditiva sensorioneural profunda na orelha esquerda.

Foram obtidos os resultados da avaliação do processamento auditivo (Tabelas 2 e 3).

Tabela 2. Escores obtidos pelo paciente em estudo nos testes monóticos do processamento auditivo

\begin{tabular}{cccc}
\hline $\begin{array}{c}\text { Testes monóticos } \\
\text { (OD) }\end{array}$ & S/R=0 & $\begin{array}{c}\text { Escore de } \\
\text { acertos }(\%)\end{array}$ & $\begin{array}{c}\text { Padrão de } \\
\text { normalidade }(\%)\end{array}$ \\
\hline \multirow{3}{*}{ SSI (ipsilateral) } & $\mathrm{S} / \mathrm{R}=-10$ & 70 & $\geq 80$ \\
& $\mathrm{~S} / \mathrm{R}=-15$ & 60 & $\geq 70$ \\
\hline Fala filtrada & & 92 & $\geq 60$ \\
\hline
\end{tabular}

Legenda: $\mathrm{S} / \mathrm{R}$ = relação sinal/ruído; $\mathrm{OD}=$ orelha direita; $\mathrm{SSI}=$ Synthetic Sentence Identification

Tabela 3. Escores obtidos pelo paciente em estudo nos testes dióticos do processamento auditivo

\begin{tabular}{lcc}
\hline Testes dióticos & $\begin{array}{c}\text { Escore de } \\
\text { acertos }\end{array}$ & $\begin{array}{c}\text { Padrão de } \\
\text { normalidade }\end{array}$ \\
\hline Localização sonora & $3 / 5(60 \%)^{*}$ & $\geq 4 / 5(80 \%)$ \\
Memória para sons verbais & $3 / 3(100 \%)$ & $\geq 2 / 3(100 \%)$ \\
Memória para sons não verbais & $3 / 3(100 \%)$ & $\geq 2 / 3(66,6 \%)$ \\
AFT-R & $6,7 \mathrm{~ms}$ & $\leq 8 \mathrm{~ms}$ \\
\hline
\end{tabular}

Legenda: AFT-R = Auditory Fusion Test - Revised; $\mathrm{ms}=$ milissegundos; ${ }^{*}=$ resultado alterado

\section{DISCUSSÃO}

A avaliação audiológica periférica convencional fornece informações básicas sobre as habilidades auditivas do sujeito. Todavia, as alterações nas habilidades auditivas utilizando medidas de avaliação comportamental da função auditiva central têm sido documentadas em sujeitos que apresentam comprometimento unilateral da audição ${ }^{(4,5)}$.

Os achados do presente caso em estudo, de um sujeito com diagnóstico de perda auditiva sensorioneural unilateral profunda à esquerda e audição dentro dos padrões de normalidade à direita, revelou alteração na habilidade de localização sonora. Essa alteração foi verificada por meio do desempenho do paciente no teste comportamental de processamento auditivo, que verifica informações sobre esta importante habilidade auditiva, corroborando um trabalho realizado, em que os indivíduos com perda auditiva unilateral apresentaram desempenho prejudicado em tarefas de localização sonora, fechamento e comunicação ${ }^{(5)}$.

A habilidade de localização sonora não é crucial apenas para determinar a origem de um som, mas também na separação de sons em um ambiente de múltiplos sons ${ }^{(9)}$. A alteração nesta habilidade era esperada ao sujeito com perda auditiva unilateral, tendo em vista que o mecanismo fisiológico auditivo de discriminação da direção da fonte sonora depende do processo de interação binaural, sendo necessária integridade auditiva bilateral. Esta interação é dependente dos sinais recebidos pelas duas orelhas e da forma como a informação auditiva é processada. Tais interações auxiliam o sujeito na localização das fontes sonoras no espaço e na realização de figura-fundo ${ }^{(9)}$.

Apesar de estudo ${ }^{(9)}$ relatar que sujeitos com dificuldade de localização sonora apresentam comprometimento na compreensão da fala em ambientes com competição de ruídos (o que requer as habilidades auditivas de fechamento e de figurafundo), tais habilidades não se encontraram alteradas no caso em estudo. Esse aspecto pôde ser verificado pelo desempenho do paciente nos testes de Fala Filtrada e SSI-escuta monótica, respectivamente. Tal achado demonstra adequação no processo de interação para o mecanismo de discriminação de sons verbais sobrepostos em escuta monótica. Este mecanismo é de fundamental importância para a compreensão da informação auditiva na presença de mensagem competitiva ${ }^{(10)}$.

No entanto, as queixas de atenção e compreensão auditiva corroboram pesquisas ${ }^{(11)}$ que afirmam que a diminuição da compreensão da fala em ambientes com competição de ruídos aumenta nos indivíduos o sentimento de confusão e perda de concentração. Restrições na execução de algumas tarefas, assim como nas atividades de interação social foram relatadas pelo sujeito do presente estudo. Tais achados concordam com os resultados de uma pesquisa ${ }^{(12)}$ em que foi avaliada a autopercepção da deficiência auditiva sensorioneural unilateral de adultos não usuários de aparelhos de amplificação sonora individual.

Diferentemente do encontrado em outro estudo ${ }^{(4)}$ utilizando o teste Gaps-in-Noise (GIN), a habilidade auditiva de resolução temporal não se mostrou alterada no presente estudo, verificada por meio do AFT-R. No entanto, os resultados aqui obtidos concordam com estudo ${ }^{(5)}$ que utilizou o RGDT (Random Gap Detection Test). Essa diferença observada entre os estudos podem ser decorrentes dos diferentes estímulos utilizados nos testes: ruído branco no teste GIN e tom puro no teste RGDT 
e no AFT-R ${ }^{(13)}$. Vale destacar que, atualmente, com o mesmo objetivo de avaliar a habilidade auditiva de resolução temporal, três testes estão disponíveis para o uso clínico (AFT-R, RGDT e o GIN).

No que se refere à habilidade auditiva de ordenação temporal, a ausência de alterações nos testes denominados TMSV e TMSnV indica que os mecanismos fisiológicos auditivos de processamento temporal se mostraram eficientes, ou seja, não são influenciados pela habilidade de localização sonora alterada e/ou pela presença de perda auditiva unilateral. No entanto, um estudo ${ }^{(8)}$ revelou relação entre o atraso da habilidade de localização sonora e a alteração do mecanismo fisiológico de processamento temporal. Isso ocorreu devido à dificuldade em lidar com pistas temporais (dificuldade em analisar pistas acústicas de tempo e intensidade), que por sua vez interferiu na habilidade em ordenar sons. Tal relação foi verificada por meio dos testes de memória para sons verbais e não verbais em sequência.

O resultado obtido neste estudo mostrando a alteração de apenas uma habilidade auditiva, dentre as cinco avaliadas, não parece ser justificado pelo lado afetado da perda auditiva. Isso porque, na presença de uma perda auditiva unilateral à direita, seria observado um impacto maior na percepção e processamento auditivo, quando comparada à perda no lado esquerdo ${ }^{(1)}$. Assim, novas pesquisas são necessárias para elucidar os mecanismos precisos que justifiquem os resultados obtidos diante da perda auditiva unilateral, tendo em vista que alterações na estimulação binaural resultam em mudanças estruturais e funcionais no sistema auditivo central ${ }^{(14)}$.

A literatura tem enfatizado os benefícios da utilização de dispositivos eletrônicos por sujeitos diagnosticados com perda auditiva, pois ela minimiza os problemas ocasionados pela privação sensorial. No entanto, neste caso em particular, devido o grau profundo da alteração auditiva e seguindo o preconizado pelo serviço ${ }^{(15)}$, não houve a indicação do uso de dispositivos eletrônicos.

\section{COMENTÁRIOS FINAIS}

Neste caso em estudo, a perda auditiva sensorioneural unilateral de grau profundo não pareceu restringir o desenvolvimento das habilidades do processamento auditivo avaliadas, exceto no que se refere à localização da fonte sonora. No entanto, foram relatadas queixas relacionadas à habilidade de localização sonora, atenção, discriminação e compreensão auditiva.

Considerando a habilidade auditiva prejudicada, bem como as queixas relatadas pelo paciente, um programa de orientação e acompanhamento da audição está sendo realizado, tendo em vista a não indicação do uso de dispositivo eletrônico, devido ao grau da perda auditiva presente. É importante salientar que este artigo se refere à apresentação e discussão de apenas um caso de perda auditiva unilateral. Portanto, os resultados e as considerações finais aqui descritos devem ser entendidos com certo cuidado, sem generalizações. Um estudo com um número maior de sujeitos deve ser conduzido para a confirmação dos achados. Além disso, faz-se necessária a realização de novas investigações, utilizando testes de processamento auditivo que avaliem outras habilidades e considerem diferentes graus da perda auditiva unilateral.

\section{REFERÊNCIAS}

1. Lieu JE. Speech-language and educational consequences of unilateral hearing loss in children. Arch Otolaryngol Head Neck Surg. 2004;130(5):524-30.

2. McKay S, Gravel JS, Tharpe A. Amplification considerations for children with minimal or mild bilateral hearing loss and unilateral hearing loss. Trends Amplif. 2008;12(1):43-54.

3. Yoshinaga-Itano C, Johnson CD, Carpenter K, Brown AS. Outcomes of children with mild bilateral hearing loss and unilateral hearing loss. Semin Hear. 2008; 29(2):196-211.

4. Vieira MR. Avaliação dos comportamentos auditivos de figura-fundo e de resolução temporal e da percepção de limitações de atividades comunicativas em crianças e adolescentes portadores de deficiência auditiva unilateral [dissertação]. São Paulo: Universidade Federal de São Paulo; 2010.

5. Nishihata R. Resolução temporal, localização e identificação de sons verbais degradados em portadores de perda auditiva unilateral com e sem dificuldade de linguagem [dissertação]. São Paulo: Universidade Federal de São Paulo; 2010

6. Pereira LD, Schochat E. Testes auditivos comportamentais para avaliação do processamento auditivo central. Barueri (SP): Pró-Fono; 2011.

7. McCrosckey R, Keith RW. AFT-R: auditory fusion test-revised. San Antonio (TX): Psychological Corporation; 1996.

8. Gallo J, Dias Kz, Pereira LD, Azevedo MF, Sousa EC. Avaliação do processamento auditivo em crianças nascidas pré-termo. J Soc Bras Fonoaudiol. 2011;23(2):95-101.

9. Takesian AE, Kotak VC, Sanes DH. Developmental hearing loss disrupts synaptic inhibition: implications for auditory processing. Future Neurol 2009;4(3):331-49.

10. Pinheiro MM, Pereira LD. Processamento auditivo em idosos: estudo da interação por meio de testes com estímulos verbais e não verbais. Rev Bras Otorrinolaringol. 2004;70(2):209-14.

11. Ruscetta MN, Palmer CV, Durrant JD, Grayhack J, Ryan C. Validity, internal consistency, and test/retest reability of a localization disabilities and handicaps questionnaire. J Am Acad Audiol. 2005;16(8):585-95.

12. Araújo PG, Mondelli MF, Lauris JR, Richiéri-Costa A, Feniman MR. Assessment of the auditory handicap in adults with unilateral hearing loss. Braz J Otorhinolaryngol. 2010;76(3):378-83.

13. Zaidan E, Garcia AP, Tedesco ML, Baran JA. Desempenho de adultos jovens normais em dois testes de resolução temporal. Pró-Fono. 2008;20(1):19-24.

14. Hutson KA, Durham D, Imig T, Tucci DL. Consequences of unilateral hearing loss: cortical adjustment to unilateral deprivation. Hear Res. 2008;237(1-2):19-31

15. Brasil. Ministério da Saúde. Política Nacional de Atenção à Saúde Auditiva. Portaria n. 2073/GM de 28 de setembro de 2004. Institui a Política Nacional de Atenção à Saúde Auditiva [Internet]. 2004 [citado 2011 Nov 10]. Disponível em: http://dtr2001.saude.gov.br/sas/ PORTARIAS/Port2004/GM/GM-2073.htm 